\title{
RELACIÓN ENTRE SÍNTOMAS PSIQUIÁTRICOS Y LA SEVERIDAD DEL USO DE DROGAS EN ESTUDIANTES UNIVERSITARIOS DE LA CIUDAD DE MONTERÍA
}

\author{
Manuel Guerrero-Martelo ${ }^{1}$ \\ Universidad Cooperativa de Colombia, Sede Montería \\ Gonzalo Daniel Galván² \\ Universidad Cooperativa de Colombia, Sede Montería \\ Universidad Nacional de la Patagonia Austral, Santa Cruz, Argentina \\ Francisco Javier Vásquez-De La Hoz ${ }^{3}$ \\ Universidad Cooperativa de Colombia, Sede Montería
}

\section{Resumen}

Existe, en los últimos años, un aumento del consumo de drogas, en especial entre los jóvenes, que cada vez consumen a más temprana edad y en mayor cantidad, esto incrementa la posibilidad de trastornos relacionados con sustancias y la comorbilidad con otros trastornos psiquiátricos. El estudio buscó describir la relación entre síntomas psiquiátricos y la gravedad de uso de drogas en jóvenes universitarios

1 Doctor en Sociología Jurídica e Instituciones Políticas. Magister en Prevención y Tratamiento de las Drogodependencias. Psicólogo. Profesor de la Facultad de Ciencias Sociales y Humanas de la Universidad Cooperativa de Colombia. Investigador Asociado, Colciencias. Email: manuel.guerrerom@campusucc.edu.co, manuelfguerrero@gmail.com

2 Doctor en Psicología, Magister en Prevención y Tratamiento de las Drogodependencias. Especialista Universitario en Hipnosis Ericksoniana. Licenciado en Psicología Orientación Clínica. Profesor de la Facultad de Ciencias Sociales y Humanas de la Universidad Cooperativa de Colombia y Universidad Nacional de la Patagonia Austral, Santa Cruz, Argentina. Investigador Senior de Colciencias. Email: danielg.galvan@campusucc.edu.co galvan. patrignani@gmail.com.

3 Magister en Educación. Especialista en Evaluación Educativa. Psicólogo. Profesor de la Facultad de Ciencias Sociales y Humanas de la Universidad Cooperativa de Colombia. Investigador Senior, Colciencias. Email: franciscoj.vasquez@campusucc.edu.co vasquezdelahoz@gmail.com. 
del departamento de Córdoba (Colombia). La muestra fue de 3550 alumnos (Varones $=48 \%$ y Mujeres $n=52 \%$ ), con edades entre los 16 y los 63 años. $(M=20.94 \mathrm{DE}=3.88)$ entre ler a 10 mo semestre. Se encontró una correlación estadísticamente significativa negativa entre el consumo de riesgo de anfetaminas y la presencia de ansiedad fóbica en hombres; en mujeres no hubo ninguna correlación significativa. No hay diferencias en el consumo de riesgo de sustancias en ambos géneros, tampoco se encuentra evidencia para la tesis de la relación existente entre síntomas psiquiátricos y consumo de sustancias, y sus implicaciones en el diagnóstico, así como tratamiento de pacientes.

Palabras clave: síntomas psiquiátricos, uso de sustancias, salud mental, universitarios, género.

\section{Abstract}

In recent years it has been observed an increase of drug use, especially among young people, who increasingly consume at an earlier age and in greater quantity, this increases the possibility of substancerelated disorders and comorbidity with other psychiatric disorders. The study sought to describe the relationship between psychiatric symptoms and the severity of drug use in university students in the department of Córdoba (Colombia). The sample was 3550 students (Men $=48 \%$ and Women $n=52 \%$ ), aged between 16 and 63 years. $(\mathrm{M}=20.94 \mathrm{SD}=3.88)$ between 1 st to 10 th semester. Finding a statistically significant negative correlation between the consumption of amphetamine risk and the presence of phobic anxiety in men; in women, there was no significant correlation. There are no differences in the consumption of risk of substances in both genders, nor is there evidence for the thesis of the relationship between psychiatric symptoms and substance use, and its implications in diagnosis, as well as treatment of patients.

Keywords: psychiatric symptoms, substance use, mental health, university, gender. 


\section{Introducción}

El aumento de consumo de drogas, así como el número de personas relacionadas con problemas de drogas, aumentó en los últimos años (United Nations Office of Drugs and Crime UNDOC, 2016) conllevando a problemas de salud mental asociados con conductas de riesgo delictivas, sexuales (Medina-Mora, et al., 2001) y comportamientos violentos (Garcia, et al., 2015). Estimaciones señalan que el $5 \%$ de la población mundial utilizó drogas en 2016 y, de este porcentaje total, el 0.6\% padece algún trastorno relacionado con el uso de sustancias (United Nations Office on Drugs and Crime, 2017).

En particular, la población de jóvenes en Colombia ha experimentado un aumento general en el consumo de sustancias, tanto licitas como ilícitas en los últimos años, con las tasas más altas de consumo de alcohol entre estudiantes de secundaria en el último mes en la región, y $65.71 \%$ de escolares que han consumido en el último año marihuana que es la sustancia ilícita más utilizada en Colombia (Observatorio de Drogas Colombia -ODC, 2017; UNDOC, 2017). El aumento en el consumo está relacionado con mayor riesgo en las consecuencias de la salud física así como mental, y un mayor riesgo determina tipos de intervenciones más complejas — para los individuos, e igualmente, para la comunidad— (Correa \& Pérez, 2013).

Cuando el uso de sustancias genera problemas de salud mental se conocen como trastornos relacionados con sustancias y aparecen frecuentemente en un mismo individuo con otros síntomas psiquiátricos o con comorbilidad psiquiátrica, hecho también conocido como patología dual (Marín-Navarrete \& Szerman, 2015; Torrens, 2008) que tiene un substrato neurobiológico (Rojas \& Castaño, 2017) y se presenta con mayor severidad en los consumidores de drogas ilícitas (Bandeira, Cordeiro, Cavalcanti, \& Duarte, 2015).

Hay una preocupación por entender la relación entre el consumo de drogas y trastornos psiquiátricos, dado que es un aspecto fundamental para el abordaje terapéutico porque permite hacer una planificación eficaz de las intervenciones (Rodriguez \& Salgado, 2017) y consecuentemente permite un direccionamiento de la estructura sanitaria disponible para ser más efectiva y eficaz (Marín-Navarrete \& Szerman, 2015). 
Entender este problema en los jóvenes es fundamental porque la edad de inicio del consumo es cada vez menor (Dörr, Gorostegui, Viani, \& Dörr, 2009; Galván, Guerrero-Martelo, \& Vásquez-De la Hoz, 2017; GuerreroMartelo, et al., 2015), lo que implica problemas de salud mental más fuertes (Hernández, et al., 2010; Van Gastel, et al., 2013) por la psicopatología asociada y otros problemas adyacentes, como por ejemplo la relación entre consumo de sustancias en jóvenes y comportamientos delictivos (RibasSiñol, et al., 2015).

Así mismo, hay una relación entre el consumo de riesgo y la aparición de síntomas psiquiátricos (Ilgen \& Moos, 2006); conocerla permite mejorar la atención ante la presencia de cualquiera de los dos. Teniendo en cuenta los resultados de Isorna, Fernandez-Ríos y Souto (2010) quienes señalan que cuando hay una coocurrencia de patología psiquiátricas y algún tipo de dependencia, el tratamiento de la dependencia tiene efectos importantes en la disminución de la severidad de la patología asociada. La relación entre trastornos psiquiátricos y consumo de riesgo en jóvenes que señala Gonzalvo, Barral, Grau-López y Esteve (2011), estudiada con mayor detalle, permitiría mejorar la atención a los pacientes de estas condiciones. Así, el consumo de riesgo y la severidad del uso de droga se encuentran en relación positiva, cuando uno aumenta el otro también.

Con base en lo anterior, este estudio tuvo como objetivo establecer la relación entre síntomas psiquiátricos y la severidad de uso de drogas en jóvenes universitarios. Esto se logró a través de los siguientes objetivos específicos:

- Determinar la prevalencia de uso de sustancias en la población escogida.

- Establecer el nivel de severidad del consumo que los usuarios de sustancias tienen.

- Evaluar la salud mental los mismos jóvenes.

- Describir la correlación entre la manifestación de síntomas psiquiátricos y la severidad del uso de sustancias de los jóvenes usuarios. 
Expuestos los objetivos de esta investigación se procede al abordaje metodológico, especificando el diseño e instrumento aplicado para dar cumplimiento a los propósitos planteados.

\section{Metodología}

En este apartado se expresan las características del diseño del estudio, los procedimientos ejecutados, asimismo el instrumento empleado a fin de alcanzar los objetivos de esta investigación.

\section{Diseño}

Es un estudio cuantitativo transversal de tipo descriptivo-correlacional (Hernández, Fernández, \& Baptista, 2014). Como estudio descriptivo describe el comportamiento de la población en torno a dos variables y sus correspondientes subescalas en un periodo de tiempo. Como estudio correlación pretende describir el tipo de relación entre las dos variables, sin buscar establecer una relación de causalidad.

\section{Procedimiento}

Con permiso de las directivas de la universidad, se hizo a la presentación del proyecto al Departamento de Bienestar Universitario. Posteriormente, los instrumentos fueron aplicados en formato virtual, explicando a los estudiantes el objetivo de la investigación y la confidencialidad de sus datos.

En conjunto con el departamento de informática se diseñó un sistema mediante el cual los estudiantes podían diligenciar los instrumentos con su cuenta personal institucional universitaria, mediante la cual aceptaban su participación. El sistema estuvo abierto aproximadamente dos semanas.

\section{Participantes}

La población fue de 15116 hombres y mujeres de un rango de 16 a 63 años, estudiantes de la Universidad de Córdoba, situada en la ciudad de Montería (Colombia) pertenecientes a diferentes carreras, que cursaban, efectivamente, el primer semestre del año 2017. 
El diseño muestral fue del tipo aleatorio no probabilístico simple. Los participantes debieron acceder a la página de la universidad desde cualquiera de los puntos de red ubicados en el campus. Se asignó un tiempo para responder las preguntas y fue hecha durante los meses de febrero y marzo de 2017. La muestra fue de 3550 alumnos de varias carreras de la universidad de Córdoba. (Varones $n=48 \%$ y Mujeres $n=52 \%$ ) de la ciudad de Montería, Colombia, con edades comprendidas entre los 16 y los 63 años de edad $(M=20.94 \mathrm{DE}=3.88)$, cursando entre 1 a 10 semestre. Se excluyeron los puntajes superiores a 63 del índice de severidad global del SCL-90-R (Casullo \& Pérez, 2008) por considerarse elevado lo que señala a una persona en alto riesgo.

\section{Instrumentos}

Para valorar las diferentes variables del estudio se utilizó un cuestionario ad hoc para datos sociodemográficos, la prueba ASSIST y el SCL-90-R.

\section{Cuestionario "ad hoc" para datos sociodemográficos.}

El instrumento ad hoc diseñado incluyó los siguientes datos: edad, sexo, situación económica, (estrato económico), lugar de origen, campus (sede de la universidad de Córdoba), status marital y carrera.

\section{Prueba de detección de consumo de alcohol, tabaco y sustancias (ASSIST).}

Diseñada con el objetivo de detectar el consumo de riesgo en torno al tabaco, alcohol y otras sustancias psicoactivas, esta prueba fue desarrollada por un conjunto de especialistas y médicos internacionales de la organización mundial de la salud (Organización Mundial de la Salud, 2011). Es un cuestionario para rellenar en papel y lápiz dirigido a consumidores de sustancias, y puede ser utilizado en varias culturas por su contenido neutral. Mide el consumo de tabaco, alcohol, cannabis, cocaína, anfetamina, inhalantes, sedantes, alucinógenos, opiáceos y otras drogas. El ASSIST indica el riesgo que corre el individuo de acuerdo al reporte de su consumo. Son tres posibles categorías de riesgo para cada sustancia: bajo medio y alto. 
Las preguntas para cada sustancia son: ¿Cuáles sustancias han consumido alguna vez a lo largo de la vida?; ¿con qué frecuencia se ha consumido sustancias en los últimos tres meses, lo que indica las sustancias más importantes para el estado de salud actual?, ¿con qué frecuencia se ha sentido un fuerte deseo o ansias de consumir cada sustancia en los últimos tres meses?, ¿con qué frecuencia el consumo de sustancias ha causado problemas en los últimos tres meses?, ¿con qué frecuencia se dejó de hacer lo que se esperaba habitualmente por el consumo de sustancias en los últimos tres meses?; ¿algún amigo, familiar o alguien más ha mostrado preocupación por los hábitos de consumo?, ¿qué tan reciente ha sido?; ¿se ha intentado alguna vez reducir o eliminar el consumo de sustancia y no se ha logrado?, ¿qué tan reciente ha sido?; ¿alguna vez ha consumido alguna droga por vía inyectada?, ¿qué tan reciente ha sido?

Cada sustancia en el cuestionario tiene un grupo de dos a siete preguntas, que tienen varias posibles respuestas y de acuerdo a cada una, una puntuación. Al final se suman las respuestas para cada sustancia específica. El puntaje puede ser entre 0 y 27 para cada sustancia, y con puntos de corte se establece en cuál de los tres niveles queda:

- Entre los 0-3 puntos indican bajo riesgo por el consumo de sustancia; no necesita intervención.

- Entre 4-26 indican riesgo moderado para consecuencias directas e indirectas del consumo de sustancias; necesita una intervención breve.

- De 27 en adelante indican alto riesgo para las consecuencias directas e indirectas del consumo de la sustancia; implica una intervención de tipo intensivo.

\section{Escala de síntomas psicopatológicos SCL-90-R:}

El SCL-90-R fue desarrollado originalmente por Leonard Derogatis para estudiar patrones de síntomas, tanto en individuos como en tareas comunitarias, este permite obtener un buen diagnóstico clínico (Sanchez $\&$ Ledesma, 2009). Consta de 90 ítems que se encuentran en escala de 0 a 4 puntos, de los cuales se obtienen resultados de 9 dimensiones y 3 índices de malestar psicológico, los cuales son: 1) Somatizaciones, 2) Obsesiones y compulsiones, 3) Sensibilidad interpersonal, 4) Depresión, 5) Ansiedad, 
6) Hostilidad, 7) Ansiedad fóbica, 8) Ideación paranoide y 9) Psicoticismo. Los tres índices son: Índice Global de severidad, índice positivo de malestar, y el Total de síntomas positivos (Casullo \& Pérez, 2008).

1. Somatizaciones: evalúa la presencia de malestares que la personal percibe relacionados con diferentes disfunciones corporales (cardiovasculares, gastrointestinales, respiratorias).

2. Obsesiones y compulsiones: incluye síntomas que se identifican con el mismo síndrome clínico (pensamientos, acciones e impulsos vivenciados como imposibles de evitar o no deseados).

3. Sensibilidad interpersonal: se focaliza en detectar la presencia de sentimientos de inferioridad e inadecuación, en especial cuando la persona se compara con sus semejantes.

4. Depresión: los ítems que componen esta subescala representan una muestra representativa de las principales manifestaciones clínicas de un trastorno depresivo: estado de ánimo disfórico, falta de motivación, poca energía vital, sentimientos de desesperanza, ideaciones y/o intentos suicidas

5. Ansiedad: evalúa la presencia de signos generales de ansiedad tales como nerviosismo, tensión, ataques de pánico, miedos.

6. Hostilidad: esta dimensión hace referencia a pensamientos, sentimientos y acciones característicos de la presencia de afectos negativos de enojo.

7. Ansiedad fóbica: este malestar alude a una respuesta persistente de miedo (a personas específicas, lugares, objetos, animales, situaciones) que en sí misma es irracional y desproporcionada en relación con el estímulo que la provoca.

8. Ideación paranoide: evalúa comportamientos paranoides fundamentalmente en tanto desordenes del pensamiento: pensamiento proyectivo, suspicacia, temor a la pérdida de autonomía.

9. Psicoticismo: incluye síntomas referidos a estados de soledad, estilo de vida esquizoide, alucinaciones y control del pensamiento.

Se suman las puntuaciones de los ítems correspondientes a cada dimensión sintomática obteniéndose una puntuación directa (PD), 
luego este resultado por la cantidad de ítems puntuados lo cual indica la puntación final. Para el caso del índice general global se suman las puntuaciones directas de todas las dimensiones y se divide por el total de

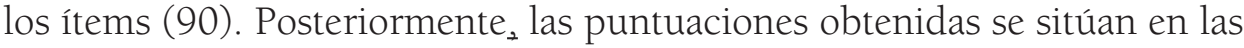
tablas de baremos y se busca la correspondencia con el percentil o bien la puntuación $t$.

Puntuaciones $t$ por debajo de 65 indican que no existe expresión sintomática de riesgo para la dimensión. Puntuaciones de 65 o más indican riesgo y puntuaciones por encima de 85 indican patología psiquiátrica severa.

La versión original del SCL-90 vino en inglés; sin embargo, se hizo la adaptación de la prueba (SCL-90-R) al español y se normalizó estadísticamente para las condiciones de la región (Casullo, 2004) y específicamente, para Colombia (Londoño et al., 2018) donde se encontraron alfas de Cronbach entre 0,74 y 0,90.

\section{Análisis de la Información}

Para realizar el análisis estadístico se utilizó el programa SPSS v .20.0, obteniendo que fueron los siguientes:

1. Datos descriptivos de las variables; a partir de los cuales se obtuvieron los descriptivos, es decir la media, los rangos y las desviaciones típicas de la edad de la muestra, así como de la puntuación global y de las 9 subescalas del SCL-90-R.

2. Se aplicó una prueba de normalidad en los resultados de las pruebas, las cuales no fueron satisfactorias. Por esta razón, fue necesario utilizar una prueba no paramétrica, en este caso la correlación de Spearman.

3. Entre las 9 subescalas del SCL-90-R y las puntuaciones de riesgo del ASSIST, se llevó a cabo una correlación de Pearson para los hombres y mujeres de la muestra por separado. 


\section{Resultados}

La Tabla 1 muestra los estadísticos descriptivos de edad y síntomas psiquiátricos de acuerdo a las puntuaciones del SCL-90-R con su índice global de puntuación y las 9 subescalas que la componen que son: 1) Psicoticismo, 2) ideación paranoide, 3) ansiedad fóbica, 4) hostilidad, 5) ansiedad, 6) depresión, 7) sensibilidad interpersonal, 8) obsesiones y compulsiones, y 9) somatizaciones, en la muestra estudiada. El mayor puntaje en general fue obtenido en la subescala de la depresión $(\mathrm{x}=10,03)$ y el menor en la subescala de la ansiedad fóbica $(x=2,20)$.

Tabla 1

Descriptivos de edad y puntuaciones de síntomas psiquiátricos*

\begin{tabular}{lccccccccccc}
\hline & Edad & IGS & Psic. & Idpa. & Anfo. & Host. & Ansi. & Depr. & Sens. & Obco. & Soma. \\
\hline Mínimo & 14,00 & 0,00 & 0,00 & 0,00 & 0,00 & 0,00 & 0,00 & 0,00 & 0,00 & 0,00 & 0,00 \\
\hline Máximo & 62,00 & 32,63 & 36,40 & 20,67 & 20,67 & 20,67 & 36,40 & 48,31 & 32,44 & 36,40 & 44,33 \\
\hline Media & 20,94 & 57,87 & 5,10 & 2,35 & 2,20 & 3,34 & 6,78 & 10,03 & 6,54 & 9,80 & 9,36 \\
\hline DT & 3,88 & 42,52 & 5,44 & 2,90 & 3,00 & 3,25 & 6,01 & 8,19 & 5,57 & 7,09 & 7,34 \\
\hline
\end{tabular}

Nota: En la puntuación y las 9 subescalas del SCL-90-R. IGS: Índice global de severidad; Psic.: Psicoticismo; Idpa.: ideación paranoide; Anfo.: Ansiedad Fóbica; Host.: Hostilidad; Ansi.: Ansiedad; Depr.: Depresión; Sens.: Sensibilidad interpersonal; Obco.: Obsesiones y compulsiones; Soma.: Somatización.

En relación a la prevalencia de uso de sustancias en los últimos tres meses puede observarse, en la Tabla 2, que las sustancias más consumidas fueron el alcohol y el tabaco, mientras las menos consumida fueron los opiáceos. No se hallaron diferencias estadísticamente significativas en cuanto al uso de sustancias entre varones y mujeres.

\section{Tabla 2}

Prevalencia de los últimos 3 meses en el consumo de sustancias por género

\begin{tabular}{llcccc}
\hline \multirow{2}{*}{ Tabaco } & & Total & Varones & Mujeres & Z \\
\hline \multirow{2}{*}{ Alcohol } & Con prevalencia (\%) & $302(8,5)$ & $150(4,2)$ & $152(4,3)$ & 0,244 \\
\cline { 2 - 5 } & Sin prevalencia (\%) & $3.248(91,5)$ & $1.565(44,1)$ & $1.683(47,4)$ & \\
\hline \multirow{2}{*}{ Cannabis } & Con prevalencia (\%) & $2.290(64,5)$ & $1.108(31,2)$ & $1.182(33,3)$ & 0,014 \\
\cline { 2 - 5 } & Sin prevalencia (\%) & $1.260(35,5)$ & $607(17,1)$ & $653(18,4)$ & \multirow{2}{*}{0,295} \\
\cline { 2 - 5 } & Con prevalencia (\%) & $96(2,7)$ & $49(1,4)$ & $47(1,3)$ & \\
\hline
\end{tabular}




\begin{tabular}{|c|c|c|c|c|c|}
\hline & & Total & Varones & Mujeres & $\mathrm{Z}$ \\
\hline \multirow{2}{*}{ Cocaína } & Con prevalencia (\%) & $11(0,3)$ & $8(0,2)$ & $3(0,1)$ & \multirow{2}{*}{2,634} \\
\hline & Sin prevalencia (\%) & $3.522(99,2)$ & $1.707(48,1)$ & $1.832(51,8)$ & \\
\hline \multirow{2}{*}{ Anfetaminas } & Con prevalencia (\%) & $11(0,3)$ & $8(0,2)$ & $3(0,1)$ & \multirow{2}{*}{2,634} \\
\hline & Sin prevalencia (\%) & $3.539(99,3)$ & $1.707(48,1)$ & $1.832(51,6)$ & \\
\hline \multirow{2}{*}{ Tranquilizantes } & Con prevalencia (\%) & $98(2,8)$ & $46(1,3)$ & $52(1,5)$ & \multirow{2}{*}{0,076} \\
\hline & Sin prevalencia (\%) & $3.452(97,2)$ & $1.669(47,0)$ & $1.783(50,2)$ & \\
\hline \multirow{2}{*}{ Inhalantes } & Con prevalencia (\%) & $15(0,4)$ & $9(0,3)$ & $6(0,2)$ & \multirow{2}{*}{0,824} \\
\hline & Sin prevalencia (\%) & $3.535(99,6)$ & $1.706(48,1)$ & $1.829(51,5)$ & \\
\hline \multirow{2}{*}{ Alucinógenos } & Con prevalencia (\%) & $10(0,3)$ & $5(0,15)$ & $5(0,15)$ & \multirow{2}{*}{0,011} \\
\hline & Sin prevalencia (\%) & $3.540(99,7)$ & $1.710(48,2)$ & $1.830(51,5)$ & \\
\hline \multirow{2}{*}{ Opiáceos } & Con prevalencia (\%) & $8(0,2)$ & $3(0,1)$ & $5(0,1)$ & \multirow{2}{*}{0,375} \\
\hline & Sin prevalencia (\%) & $3.542(99,8)$ & $1.712(48,2)$ & $1.8301,7)$ & \\
\hline
\end{tabular}

La Tabla 3 presenta los descriptivos de las puntuaciones de gravedad de uso de drogas para cada sustancia. La máxima puntuación de riesgo fue para el alcohol y la mínima para los opiáceos.

Tabla 3

Descriptivos de las puntuaciones de gravedad de uso de sustancias

\begin{tabular}{lcccc}
\hline & Mínimo & Máxima & Media & DT \\
\hline Tabaco & $<0,0001$ & 36 & 0,97 & 3,78 \\
\hline Alcohol & $<0,0001$ & 33 & 4,24 & 5,45 \\
\hline Cannabis & $<0,0001$ & 33 & 0,27 & 2,15 \\
\hline Cocaína & $<0,0001$ & 33 & 0,066 & 1,22 \\
\hline Anfetamina & $<0,0001$ & 33 & 0,074 & 1,17 \\
\hline Inhalantes & $<0,0001$ & 33 & 0,057 & 1,06 \\
\hline Tranquilizantes & $<0,0001$ & 36 & 0,30 & 2,15 \\
\hline Alucinógenos & $<0,0001$ & 33 & 0,045 & 1 \\
\hline Opiáceos & $<0,0001$ & 33 & 0,032 & 0,87 \\
\hline
\end{tabular}

En la relación entre la puntuación de riesgo de las nueve sustancias que forman parte del ASSIST y su correlación con los puntajes del SCL90-R, y sus correspondientes subescalas para las mujeres de la muestra mediante el coeficiente de Spearman, se encontró que no existe ninguna correlación estadísticamente significativa (véase la Tabla 4). 
Relación entre síntomas psiquiátricos y la severidad del uso de drogas en estudiantes universitarios de la ciudad de Montería

\section{Tabla 4}

Relación entre puntuación de riesgo de sustancias y síntomas psiquiátricos en mujeres

\begin{tabular}{|c|c|c|c|c|c|c|c|c|c|c|c|c|c|c|c|c|c|c|c|}
\hline & 1 & 2 & 3 & 4 & 5 & 6 & 7 & 8 & 9 & 10 & 11 & 12 & 13 & 14 & 15 & 16 & 17 & 18 & 19 \\
\hline $\begin{array}{l}\text { 1-Riesgo } \\
\text { consumo de } \\
\text { tabaco }\end{array}$ & 1,000 &, $244^{\text {*t }}$ &, $304^{* *}$ &, $177^{* *}$ &, $088^{\star *}$ &, $051^{*}$ &, $128^{\star \star}$ &, $102^{* *}$ &, $114^{* *}$ & ,019 & ,040 & ,039 & ,036 & ,036 & ,011 & ,027 & ,034 & ,025 & ,032 \\
\hline $\begin{array}{l}\text { 2-Riesgo } \\
\text { consumo de } \\
\text { alcohol }\end{array}$ & & 1,000 &, $078^{* *}$ &, $069^{* *}$ &, $067^{+*}$ & ,040 &, $080^{* *}$ &, $061^{*}$ &, $053^{*}$ &,- 013 &,- 016 &,- 007 &,- 014 &,- 017 &,- 028 &,- 036 &,- 018 &,- 022 &,- 020 \\
\hline $\begin{array}{l}\text { 3-Riesgo } \\
\text { consumo de } \\
\text { cannabis }\end{array}$ & & & 1,000 &, $262^{* *}$ &, $246^{\star *}$ & $118^{* *}$ &, $135^{\star *}$ & $355^{* *}$ &, $231^{\star *}$ & ,022 & ,017 & ,011 &,- 013 &,- 003 &,- 031 &,- 008 & ,012 &,- 002 & ,000 \\
\hline $\begin{array}{l}\text { 4-Riesgo } \\
\text { consumo de } \\
\text { cocaína }\end{array}$ & & & & 1,000 &, $433^{* *}$ &, $353^{*}$ &, $191^{\star *}$ &, $613^{* *}$ &, $447^{+*}$ & ,007 & ,008 & ,012 & ,010 & ,020 & ,004 & ,001 & ,006 & ,007 & ,012 \\
\hline $\begin{array}{l}\text { 5-Riesgo } \\
\text { consumo de } \\
\text { anfetaminas }\end{array}$ & & & & & 1,000 & ,201" &, $215^{\star \star}$ & ,589* &, $257^{\star \star}$ & ,031 & ,016 & ,004 &,- 009 &, 004 & ,009 &,- 008 & ,021 & ,009 & ,010 \\
\hline $\begin{array}{l}\text { 6-Riesgo } \\
\text { consumo de } \\
\text { inhalantes }\end{array}$ & & & & & & 1,000 &, $129^{* *}$ &, $288^{* *}$ &, $315^{* *}$ &,- 030 &,- 002 &,- 011 &,- 033 &,- 020 &,- 033 &,- 017 &,- 017 &,- 005 &,- 023 \\
\hline $\begin{array}{l}\text { 7-Riesgo } \\
\text { consumo de } \\
\text { tranquilizante } \\
\mathrm{s}\end{array}$ & & & & & & & 1,000 & ,206* &, $110^{* *}$ & ,005 & ,020 &, 006 & ,014 &, 022 &, 011 &,- 022 & ,016 & ,005 &, 012 \\
\hline $\begin{array}{l}\text { 8-Riesgo } \\
\text { consumo de } \\
\text { alucinógenos }\end{array}$ & & & & & & & & 1,000 &, $365^{\star *}$ & ,013 & ,003 &,- 003 &,- 007 &,- 009 &, 005 & ,013 & ,018 & ,010 & ,000 \\
\hline $\begin{array}{l}\text { 9-Riesgo } \\
\text { consumo de } \\
\text { opiaceos }\end{array}$ & & & & & & & & & 1,000 &,- 003 & ,011 & ,015 & ,009 &, 006 &,- 023 & ,004 & ,000 & ,018 & ,002 \\
\hline $\begin{array}{l}10- \\
\text { Somatizacion } \\
\text { es }\end{array}$ & & & & & & & & & & 1,000 &, $711^{* *}$ &, $659^{* *}$ &, $732^{* *}$ &, $766^{\star \star}$ & $629^{* *}$ &, $575^{* *}$ & $675^{\star \star}$ & ,689* &, $856^{\star *}$ \\
\hline $\begin{array}{l}11- \\
\text { Obsesiones } \\
\text { y } \\
\text { compulsione } \\
\text { s }\end{array}$ & & & & & & & & & & & 1,000 &, $779^{\star *}$ & $818^{* *}$ &, $773^{\star *}$ &, $618^{\star *}$ &, $574^{* *}$ &, $738^{* *}$ &, $758^{* *}$ & $899^{* \star}$ \\
\hline $\begin{array}{l}12- \\
\text { Sensitividad } \\
\text { interpersonal }\end{array}$ & & & & & & & & & & & & 1,000 &, $813^{* *}$ &, $760^{\star \star}$ &, $627^{\star *}$ &, $613^{* *}$ &, $798^{\star *}$ & $810^{* *}$ &, $879^{* *}$ \\
\hline $\begin{array}{l}\text { 13- } \\
\text { Depresión }\end{array}$ & & & & & & & & & & & & & 1,000 &, 776 "* &, $667^{+*}$ &, $591^{* *}$ &, $780^{* *}$ &, $797^{* *}$ &, $923^{* *}$ \\
\hline 14-Ansiedad & & & & & & & & & & & & & & 1,000 &, $635^{\star *}$ &, $674^{* *}$ &, $738^{* *}$ &, $780^{* *}$ &, $894^{\star \star}$ \\
\hline 15-Hostilidad & & & & & & & & & & & & & & & 1,000 &, $497^{* *}$ &, $669^{* *}$ &, $645^{* *}$ &, $746^{* *}$ \\
\hline $\begin{array}{l}16-\text { Ansiedad } \\
\text { fóbica }\end{array}$ & & & & & & & & & & & & & & & & 1,000 &, $594^{\star *}$ &, $637^{* *}$ & ,695 \\
\hline $\begin{array}{l}\text { 17-Ideación } \\
\text { paranoide }\end{array}$ & & & & & & & & & & & & & & & & & 1,000 &, 796 &, $861^{* *}$ \\
\hline $\begin{array}{l}\text { 18- } \\
\text { Psicoticismo }\end{array}$ & & & & & & & & & & & & & & & & & & 1,000 &, $861^{\star \star}$ \\
\hline $\begin{array}{l}\text { 19-Indice } \\
\text { Global SCL- } \\
90\end{array}$ & & & & & & & & & & & & & & & & & & & 1,000 \\
\hline
\end{tabular}




\section{Tabla 5}

Relación entre puntuación de reisgo de consumo se sustacias y síntomas psiquiátricos en hombres

\begin{tabular}{|c|c|c|c|c|c|c|c|c|c|c|c|c|c|c|c|c|c|c|c|}
\hline & 1 & 2 & 3 & 4 & 5 & 6 & 7 & 8 & 9 & 10 & 11 & 12 & 13 & 14 & 15 & 16 & 17 & 18 & 19 \\
\hline $\begin{array}{l}\text { 1-Riesgo } \\
\text { consumo de } \\
\text { tabaco }\end{array}$ & 1,000 &, $259^{* *}$ &, $377^{* t}$ &, $220^{\text {* }}$ & $175^{\star *}$ & $155^{\star *}$ &, $160^{* *}$ &, $099^{\text {kt }}$ &, $070^{* *}$ & ,000 & ,023 & ,006 &,- 002 &,- 006 &,- 027 &,- 034 & ,000 & ,020 & ,003 \\
\hline $\begin{array}{l}\text { 2-Riesgo } \\
\text { consumo de } \\
\text { alcohol }\end{array}$ & & 1,000 &, $134^{* *}$ & ,106 & ,108** & ,091" & ,090 &, $061^{*}$ &, $075^{\star *}$ &,- 009 & ,013 & ,021 & ,012 & ,017 & ,012 & ,008 & ,014 & ,026 & ,014 \\
\hline $\begin{array}{l}\text { 3-Riesgo } \\
\text { consumo de } \\
\text { cannabis }\end{array}$ & & & 1,000 &, $392^{* *}$ &, $361^{* *}$ &, $265^{* *}$ &, $147^{* *}$ &, 206 ** &, $150^{* *}$ &,- 011 & ,004 & ,025 &,- 008 & ,002 &,- 007 &,- 040 & ,001 & ,019 & ,002 \\
\hline $\begin{array}{l}\text { 4-Riesgo } \\
\text { consumo de } \\
\text { cocaína }\end{array}$ & & & & 1,000 &, $614^{* *}$ &, $271^{* *}$ &, $110^{* *}$ &, $234^{* *}$ &, 386 & ,008 &,- 006 & ,006 &,- 008 & ,001 &,- 015 &,- 030 & ,006 &,- 001 & ,003 \\
\hline $\begin{array}{l}\text { 5-Riesgo } \\
\text { consumo de } \\
\text { anfetaminas }\end{array}$ & & & & & 1,000 &, $250^{* *}$ &, $175^{* *}$ &, $216^{* *}$ & ,238** &,- 022 &,- 015 &,- 032 &,- 016 &,- 029 &,- 022 &,$- 058^{*}$ &,- 013 &,- 028 &,- 021 \\
\hline $\begin{array}{l}\text { 6-Riesgo } \\
\text { consumo de } \\
\text { inhalantes }\end{array}$ & & & & & & 1,000 &, $121^{* *}$ &, $257^{* *}$ &, $282^{* *}$ &,- 025 &,- 026 &,- 008 &,- 015 &,- 037 &,- 010 &,- 016 &,- 030 &,- 004 &,- 023 \\
\hline $\begin{array}{l}\text { 7-Riesgo } \\
\text { consumo de } \\
\text { tranquilizante } \\
\mathrm{S}\end{array}$ & & & & & & & 1,000 &, $165^{* *}$ &, $119^{* *}$ & ,032 & ,011 & ,008 &,- 011 & ,004 & ,023 & ,016 & ,017 & ,015 & ,015 \\
\hline $\begin{array}{l}\text { 8-Riesgo } \\
\text { consumo de } \\
\text { alucinógenos }\end{array}$ & & & & & & & & 1,000 &, $365^{* *}$ &,- 017 &,- 018 &,- 015 &,- 025 &,- 029 &,- 017 &,- 004 &,- 035 &,- 025 &,- 023 \\
\hline $\begin{array}{l}\text { 9-Riesgo } \\
\text { consumo de } \\
\text { opiaceos }\end{array}$ & & & & & & & & & 1,000 & 007 &, 003 & ,000 &,- 006 &,- 006 &,- 009 &,- 018 &,- 025 &,- 024 &,- 002 \\
\hline $\begin{array}{l}10- \\
\text { Somatizacion } \\
\text { es }\end{array}$ & & & & & & & & & & 1,000 &, $730^{* *}$ &, $682^{* *}$ &, $753^{* *}$ &, $777^{* *}$ &, $670^{* *}$ &, $586^{* *}$ &, $688^{* *}$ &, $699^{* *}$ &, $859^{* *}$ \\
\hline $\begin{array}{l}11- \\
\text { Obsesiones y } \\
\text { compulsione } \\
\text { s }\end{array}$ & & & & & & & & & & & 1,000 &, $783^{* *}$ &, $841^{* *}$ &, $790^{* *}$ &, $665^{\star *}$ &, $551^{* *}$ &, $757^{\star *}$ &, $754^{* *}$ &, $911^{* *}$ \\
\hline $\begin{array}{l}12- \\
\text { Sensitividad } \\
\text { interpersonal }\end{array}$ & & & & & & & & & & & & 1,000 &, $829^{* *}$ &, $781^{*}$ &, $668^{* *}$ &, $612^{* *}$ &, $804^{* *}$ &, $799^{* *}$ &, $885^{* *}$ \\
\hline 13-Depresión & & & & & & & & & & & & & 1,000 &, $810^{* *}$ &, $721^{* *}$ &, $620^{* *}$ &, $798^{* *}$ &, $816^{* *}$ &, $935^{* *}$ \\
\hline 14-Ansiedad & & & & & & & & & & & & & & 1,000 &, $680^{* *}$ &, $663^{* *}$ &, $759^{* *}$ &, $799^{* *}$ &, $901^{* *}$ \\
\hline 15-Hostilidad & & & & & & & & & & & & & & & 1,000 &, $534^{* *}$ &, $696^{* *}$ &, $666^{* *}$ &, $789^{* *}$ \\
\hline $\begin{array}{l}\text { 16-Ansiedad } \\
\text { fóbica }\end{array}$ & & & & & & & & & & & & & & & & 1,000 &, $598^{* *}$ &, $638^{* *}$ & ,678* \\
\hline $\begin{array}{l}\text { 17-Ideación } \\
\text { paranoide }\end{array}$ & & & & & & & & & & & & & & & & & 1,000 &, $799^{* *}$ & ,869* \\
\hline $\begin{array}{l}\text { 18- } \\
\text { Psicoticismo }\end{array}$ & & & & & & & & & & & & & & & & & & 1,000 & ,859** \\
\hline $\begin{array}{l}\text { 19-Indice } \\
\text { Global SCL- } \\
90\end{array}$ & & & & & & & & & & & & & & & & & & & 1,000 \\
\hline
\end{tabular}

La Tabla 5 señala las correlaciones de los hombres que formaban parte de la muestra entre la puntuación de riesgo del ASSIST y las puntuaciones obtenidas en el índice global y las 9 subescalas del SCL-90-R. Solo se encontró una correlación negativa significativa (Rho $=-0,58$ ) entre la subescala ansiedad fóbica y la puntuación de riesgo de las anfetaminas. Un Rho de Spearman de entre 0,40 y 0,59 se considera una correlación moderada. 


\section{Discusión}

La prevalencia de sustancias en los últimos tres meses de la muestra señala que las sustancias más utilizadas son: alcohol, tabaco, tranquilizantes y cannabis. Separado por género, los datos se mantienen, pero los hombres muestran mayor inclinación al uso de cannabis y las mujeres a los tranquilizantes.

Tanto en hombres como en mujeres - teniendo en cuenta que no todos los integrantes de la muestra consumen sustancias, y que tampoco todas los que consumen sustancias tienen un síntoma psiquiátrico-, se confirma lo encontrado en otros estudios que señalan altas puntuaciones en las subescalas del SCL-90-R e individuos con dependencia a sustancias (López \& Becoña, 2006; Rodríguez \& Salgado, 2017). Sin embargo, en este estudio solo se confirma una relación consistente entre el consumo de riesgo de las anfetaminas y la ansiedad fóbica como subescala del SCL-90-R en hombres.

Los resultados de las mujeres no señalan ninguna correlación significativa entre síntomas psiquiátricos y puntuaciones de riesgo de consumo de sustancias. También se encontró que no hay diferencias significativas de consumo entre hombres y mujeres, por lo cual adquiere sentido el hecho de que no hay correlación significativa con las subescalas del SCL-90-R, exceptuando la de la ansiedad fóbica y consumo de anfetaminas para los hombres.

A mayor uso de anfetaminas, menor ansiedad fóbica. Teniendo en cuenta las condiciones culturales de la muestra, tiene sentido, en tanto que la respuesta persistente de miedo para los hombres baja con el uso de anfetaminas. Un estudio señala que las anfetaminas pueden aumentar la sociabilidad porque decrementan la sensibilidad a las expresiones emocionales sutiles (Wardle, Garner, Munafo, \& de Wit, 2012), lo cual le permitiría a los hombres jóvenes universitarios de Montería menos sensibilidad para reconocer expresiones, y con esto disminuir el miedo a los eventos sociales que pueden significar incertidumbre, dado que las expresiones faciales de otros hombres pueden ser reconocidas como peligrosas, por efecto de los valores que inculcan en la región, donde nunca están tristes y siempre deben exhibir control, y mostrar su posición frente a otros hombres. 
Entre otros de los comportamientos tradicionales de la región exigidos para los hombres esta la no expresión de sus emociones y el hecho de no reconocer la expresión de los demás puede ampliar el deseo de hablar y su sociabilidad. El estudio de Bedi, Hyman y de Wit (2010) demuestra que el consumo de anfetamina reduce la identificación de posibles señales faciales emocionales, lo cual incrementa el comportamiento de aproximación social y quizás pueda ser útil a nivel de psicoterapia. Como señala otro estudio, las anfetaminas pueden tener un valor terapéutico para el tratamiento de los desórdenes de ansiedad al proveer un balance entre la activación de emociones y el sentimiento de seguridad y control (Johansen $\&$ Krebs, 2009).

Según el boletín de salud de Colombia, la ansiedad Fóbica es uno de los trastornos que más ha aumentado en los adolescentes en el periodo del 2009 al 2017- (Minsalud, 2018). Desde el modelo de la automedicación (Khantzian, 1997; West, 2005; Bolton, Cox, Clara, \& Sareen, 2006) puede tener sentido que los hombres busquen manejar el miedo persistente con las anfetaminas, en tanto que estas producen sensación de placer (Bedi et al., 2010).

\section{Conclusiones}

La evidencia parece señalar que, teniendo hombres y mujeres con las mismas características sociodemográficas, no difieren en el tipo de sustancias que consumen, sino en el uso que les pueden otorgar, en este caso los hombres perciben un decremento en un síntoma mental por efecto del consumo de una sustancia ilegal, como posible forma de automedicación.

Así mismo, se contradice la evidencia que señala una relación existente entre síntomas psiquiátricos y consumo de sustancias, así como sus implicaciones en el diagnóstico y tratamiento de pacientes, pues se encontró que las anfetaminas, cuyo uso terapéutico ha sido propuesto por expertos, puedo estar siendo usada por los hombres para controlar un miedo persistente relacionado con las condiciones culturales de la región.

Es necesaria hacer más investigaciones para corroborar lo encontrado en el estudio y seguir investigando en esta línea pues algunas teorías 
sugieren que se debe abandonar la clásica hipótesis de la automedicación, idea expuesta en el desarrollo de este trabajo investigativo.

\section{Referencias}

Bandeira, M., Cordeiro, S., Cavalcanti, M., \& Duarte, M. (2015). Presence of dual diagnoses between users and non-users of licit and ilicit drugs in Brazil. Journal Brasil do Psiquiatria, 64(4), 288-295. doi:DOI: 10.1590/0047-2085000000091.

Bedi, G., Hyman, D., \& de Wit, H. (2010). Is ecstasy an "empathogen"? Effects of MDMA on prosocial feelings and identification of emotional states in others. Biological psychiatry, 68(12), 1134-1140.

Bolton, J., Cox, B., Clara, I., \& Sareen, J. (2006). Use of alcohol and drugs to self-medicate anxiety disorders in a nationally representative sample. The journal of Nervous and Mental Disease, 194(11), 818825. doi:10.1097/01.nmd.0000244481.63148.98.

Casullo, M. (2004). Síntomas psicopatológicos en adultos urbanos. Psicología y ciencia social, 6(1), 49-57.

Casullo, M., \& Pérez, M. (2008). El inventario de sintomas SCL-90-R de L. Derogatis. Buenos Aires: UBA-CONICET.

Correa, A., \& Pérez, A. (2013). Relación e impacto de consumo de sustancias psicoactivas sobre la salud en Colombia. Liberabit, 19(2), 153-162.

Dörr, A., Gorostegui, M., Viani, S., \& Dörr, M. (2009). Adolescentes consumidores de marihuana: implicaciones para la familia y la escuela. Salud Mental, 32, 269-278.

Galván, G., Guerrero-Martelo, M., \& Vásquez-De la Hoz, F. (2017). Canabis: una ilusión cognitiva. Revista Colombiana de Psiquiatría, 55126. doi:10.1016/j.rcp.2016.04.002.

Garcia, H., Ferreira de Oliveira, M., Titus, J., Lopes, I., Hayasi, P., \& Ribeiro, R. (2015). Uso de drogas, salud mental y problemas relacionados con el crimen y la violencia: estudio transversal. Rev. Latino-Am. Enfermagem, 23(6), 1173-1180. doi:DOI: 10.1590/01041169.0478 .2663$.

Gonzalvo, B., Barral, L., Grau-López, O., \& Esteve, C. (2011). Comorbilidad psiquiátrica en adolescentes consumidores de cannabis. Trastornos adictivos, 13(3), 109-112. 
Guerrero-Martelo, M., Galván, G., Pinedo, J., Vásquez De la Hoz, F., Torres, F., \& Torres, J. (2015). Prevalencia de vida de uso de cannabis y rendimiento académico en adolescentes. Salud Uninorte, 467-478.

Hernández, R., Fernández, C., \& Baptista, M. (2014). Metodología de la investigación. México: Mac Graw Hill.

Hernández, T., Roldán, J., Jiménez, A., Mora, C., Sánchez-Garnica, D., \& Pérez, M. (2010). Age at initiation of drug use as an indicator for problematic use. Psychology in Spain, 14(1), 22-31.

Ilgen, M., \& Moos, R. (2006). Exacerbation of psychiatric symtomps during substance use desorder treatment. Psychiatric services, 57(12), 1758-1764.

Isorna, M., Fernandez-Rios, L., \& Souto, A. (2010). Treatment of drug adicction and psychopathology: a field study. The European Journal of Psychology Applied to Legal Context, 2(1), 3-18.

Johansen, P., \& Krebs, T. (2009). How could MDMA (ecstasy) help anxiety disorders? A neurobiological rationale. Journal of psychofarmacology, 23(4), 389-391.

Khantzian, E. (1997). The self-medication hypothesis of substance use disorders: a reconsideration and recent applications. Harvard Review of Psychiatry, 4(5), 231-244. doi:10.3109/10673229709030550.

Londoño, N., Agudelo, D., Martínez, E., Anguila, D., Aguirre, D., \& Arias, J. (2018). Validación del cuestionario de 90 síntomas SCL-90-R de Derogatis en una muestra colombiana. Medunab, 21(2), 45-59.

López, A., \& Becoña, E. (2006). Consumo de cocaína y psicopatología asociada: una revisión. Adicciones, 18(2), 161-196.

Marín-Navarrete, R., \& Szerman, N. (2015). Repensando el concepto de adicciones: pasos hacia la patología dual. Salud Mental, 38(4), 395396. doi:DOI: 10.17711/SM.0185-3325.2015.060.

Medina-Mora, M., Natera, G., Borges, G., Cravioto, P., Fleiz, C., \& Tapia, R. (2001). Del siglo XX al tercer milenio. Las adicciones y la salud pública: drogas, alcohol y sociedad. Salud Mental, 24(4), 3-19.

Minsalud. (2018). Boletín de salud mental. Salud mental en niños, niñas y adolescentes. Bogotá: Minsalud.

Observatorio de Drogas Colombia (ODC). (2017). Reporte de drogas de Colombia 2017. Bogotá: Minjusticia-ODC. 
Organización Mundial de la Salud. (2011). La prueba de detección de consumo de alcohol, tabaco y sustancias (ASSIST) - Manuel para uso en la atención primaria. Organización Mundial de la Salud.

Ribas-Siñol, M., Del Prado-Sánchez, N., Claramunt-Mendoza, J., Civit-Ramirez, M., Canalias-Perez, O., \& Ochoa, S. (2015). Adolescentes multiproblemáticos: consumo de tóxicos y trastorno mental en jóvenes que delinquen. Actas Esp. Psiquiatr, 43(6), 197-204.

Rodríguez, J., \& Salgado, A. (2017). Patología dual en dogodependientes en tratamiento: resultados con el SCL-90-R. Revista Española de Drogodependencias, 42(3), 46-56.

Rojas, L., \& Castaño, G. (2017). Neurobiología de la patología dual. Health and Addictions, 17(2), 101-114.

Sanchez, R., \& Ledesma, R. (2009). Análisis psicométrico del inventario de síntomas revisado (SCL-90-R) en población clínica. Revista Argentina de Clínica Psicológica, 18, 265-274.

Torrens, M. (2008). Patología dual: situación actual y retos de futuro. Adicciones, 20(4), 315-320.

UNDOC. (2016). World drug report 2016. New York: United nations.

UNDOC. (2017). III estudio Epidemiológico Andino sobre Consumo de Drogas en la Población Universitaria de Colombia, 2016. Oficina de las Naciones Unidas contra la Droga y el Delito.

United Nations Office on Drugs and Crime. (2017). World Drug Report. United Nations.

Van Gastel, W. A., Tempelaar, W. M., Bun, C. J., Schubert, C. D., Kahn, R. S., Plevier, C. M., \& Boks, M. P. (2013). Cannabis use as an indicator of risk for mental health problems in adolescents: a population-based study at secondary schools. Psychological Medicine Journal, 43(9), 1849 - 1856. doi:10.1017/S0033291712002723

Wardle, M., Garner, M., Munafo, M., \& de Wit, H. (2012). Amphetamine as a social drug: effects od d-amphetamine on social processing and behaviour. Psychofarmacology, 223(2), 199-210.

West, R. (2005). Theory of addiction. Londres: Blackwell publishing. 